\title{
Comprehensive Psychiatry
}

(Official Journal of the American Psychopathological Association)

VOL. 29, NO. 1

JANUARY/FEBRUARY 1988

\section{The Daily Distribution of Panic Attacks}

\author{
George N.M. Gurguis, Oliver G. Camerom, William A. Ericson, \\ and George $C$. Curtis
}

The daily distribution of anxiety attacks was determined for seven consecutive days in 69 drug-free people with DSM-III defined panic attacks. Unexpected full symptom attacks (three or more symptoms) tended to cluster across days (i.e., were not completely random),

(-) 1988 by Grune \& Stratton, Inc.

\begin{abstract}
A. SINE QUA NON for the diagnosis of panic attacks by DSM-III or A DSM-III-R is the unexpected occurrence of at least some attacks. However, there is almost no systematic data concerning whether the distribution of panic attacks is random (i.e., unexpected because unpredictable in principle). Uhde et al. ${ }^{1}$ claim that the altacks they studied seemed to occur in random patterns, although waves of attacks occurred rarely; however, they provided no data. Cameron et al. ${ }^{2}$ and Taylor et al. ${ }^{3}$ recorded the occurrence of symptoms in panic patients. Cameron et $a .^{2}$ reported a tendency for symptoms to be greater in the afternoon or evening while Taylor et $a .^{3}$ did not report any circadian pattern. No study has examined whether attacks tend to cluster across days; that is the purpose of this report.
\end{abstract}

\section{METHOD}

Sixty-nine patients 18 to 60 years of age who met DSM-III criteria for panic disorder or agoraphobia with panic attacks participated; all gave informed consent. All patients were diagnosed with the Structured Clinical Interview for DSM-III-Upjohn Version (SCID-UP), and all were medically healthy and drug free for at least seven days prior to study. Additionally, all medications used within the previous 2 months were recorded, including which drugs were used, and when they were discontirued. All had had at least one attack per weck for the 3 weeks immediately prior to the study. In order to determine the distribution of attacks, all patients recorded in a written diary each occurrence of each of four different kinds of attacks each day for 1 week while drug free. These attack types were (a) situationally-provoked, and (b) unexpected full panic attacks (3 or more symptoms from the DSM-III Ist), and (c) situationally-provoked and (d) unexpected limited-symptom attacks ( 1 or 2 symptoms).

Statistical analyses of the data were based on the notion that: Given that a subject experienced $n$

From the Department of Psychiatry, and the Department of Statistics and Statistical Research Laboratory, University of Michigan, Ann Arbor.

This research was part of the Xanax Cross-National Panic Study. Second Phase, and was supported in part by the Upjohn Pharmaceutical Company, Kalamazoo, MT.

Address reprint requests to Oliver G. Cameron, M.D., Ph.D., Department of Psychiatry, University of Michigan Medical Center, 1500 E Medical Center Dr, Ann Arbor, MI 48109.

(0) 1988 by Grune \& Stratton, Inc.

$0010-440 X / 88 / 2901 / 0001 \$ 03.00 / 0$ 
attacks during the week, then the distribution of these among the seven days may be likened to a classical occupancy problem in probability (pp. 38-42). ${ }^{4}$ In particular, under the hypothesis of randomness, it was assumed that the joint distribution of the number of the $n$ attacks occurring on each of the seven days was multinomial with all cell probabilities equal to $1 / \%$, i.e., the so-called Maxwell-Boltzmann model. A simple $\chi^{2}$ test of the goodness of fit of this model could not be implemented because the n's differed from subject to subject and because the number of possible multinomial outcomes was far too large for most of the n's observed. To bypass this difficulty for each subject, $\mathrm{m}$ (= the number of the seven days which were attack-free) as the basis of a test statistic was used. Given the Maxwell-Boltzmann model of randomness, the probability distribution of $\mathrm{m}$ is known, (p. 60), ${ }^{4}$ and clearly depends on $\mathrm{n}$. Again, simple $\chi^{2}$ tests of the goodness of fit of these distributions could not be justified because of the large number of different $n$ 's and the few subjects having each $\mathrm{n}$. Instead, the following approach was adopted. The probability distribution of $m$ was computed for each observed value of $n$; then each $(m, n)$ pair was classified as "small" if the probability of $\mathrm{m}$ given $\mathrm{n}$ was $\leq .25$ and $\mathrm{m}$ was below the median or mode $(\mathrm{m}, \mathrm{n})$, was classified as "large" if its conditional probability was $\leq .15$ and $\mathrm{m}$ was above the median or mode, otherwise $(\mathrm{m}, \mathrm{n})$ was classified as "not large or small." The observed and expected frequencies were computed for each of these three categories and each $\mathbf{n}$ was then summed over all observed values of $\mathbf{n}>$ 1. The discrepancies between the observed and expected frequencies were then assessed using $\chi^{2}$ tests on two degrees of freedom $(d f)$. The .25 and .15 cut points defining the three classes were the smallest possible values needed to obtain large enough expected cell frequencies to justify application of these $\chi^{2}$ tests. Thus, the $\chi^{2}$ test was used to determine if the observed number of attack-free days agreed with the model (i.e., random distribution of attacks), was too large (i.e., clustering of attacks on a few days, with too many attack-free days), or was too small. Separate tests were performed for the four types of attacks described above, and also for three groupings of attacks-all unexpected and all situational (limited plus full symptom), and all full symptom attacks (unexpected plus situational).

Simply stated, the statistical analysis consisted of determining the total number of attacks and the number of days on which no attacks occurred. The number of attack-free days was compared to the expected number of attack-free days, given the total number of attacks and assuming random distribution of these attacks. Too many attack-free days would imply "clumping" of attacks, while too few would indicate a nonrandom "flattening" of the distribution.

\section{RESULTS}

The distribution of all four types of attacks across consecutive days 1 to 7 of the study did not differ significantly from equal frequencies. The average number of unexpected full panic attacks per week per patient was 3.45 (238 unexpected full attacks in 69 paticnts). The averages for the other three types were 3.64 (251 full situational attacks), 4.68 (323 limited situational attacks), and 3.67 (253 limited unexpected attacks).

Fifty-five percent of the subjects had taken some psychotropic medication within 2 months prior to the drug-free study period. Almost all of them had received a benzodiazepine. Because withdrawal effects might influence the attack distribution, patients who had taken medication were compared to those who had not. No difference was found either in the total number of attacks or the number of attack-free days between the two groups.

The $\chi^{2}$ values for the four types of attacks were 7.75 (situational full), 4.85 (situational limited), 8.48 (unexpected full), and 1.16 (unexpected limited). With 2 $d f$, statistical tests for both types of full attacks were significant $\left(\chi^{2}\right.$ of 7.38 required for $P=.025$ ). For all four types of attacks, the expected number of attack-free days were greater than expected, for both the small and large cells; however, in each of the four cases, the large class made the biggest contribution to the $\chi^{2}$ statistic. This result implies that clustering of attacks did occur.

For the three combined groups (all full, all situational, all unexpected), the data also indicated clustering, but none of the $\chi^{2}$ tests were significant. For all 
unexpected attacks, a trend was observed $\left(\chi^{2}=4.19,2 d f, P=.123\right)$. Thus, there appears to be some evidence of clustering, especially for the unexpected full symptom attacks.

\section{DISCUSSION}

Even with this relatively short duration of study (seven days), evidence for clustering, especially of full unexpected attacks, was observed. Longer duration studies might show an even stronger effect.

Although a tendency for clustering of situational panic attacks, especially full attacks, was also observed, it was not as strong as for the unexpected attacks. Situational anxiety is at least partially under the person's control; people with agoraphobia with panic attacks may demonstrate a clustering of avoidance behavior (i.e., agoraphobic symptoms) rather than a clustering of situational panic attacks, which might, in turn, be in phase with the clustering of unexpected attacks. This hypothesis will require further study.

If unexpected attacks do indeed cluster, what might be the mechanism? Unexpected ("spontaneous") panic attacks seem to be due at least in part to physiological abnormalities. ${ }^{5,6}$ These acute brief episodic attacks have been likened to "seizures"; retrospective reports of anxious patients indicate an increased likelihood of seizures $(3.7 \%),{ }^{7}$ and epilepsy is sometimes associated with anxiety symptoms. ${ }^{1,8,9}$ Also, seizure susceptibility sometimes shows kindling-a tendency for one seizure-like event to increase the likelihood of another. ${ }^{10}$ It is possible that panic attacks also demonstrate kindling, leading to clustering of attacks. Alternatively, the psychological (cognitive) reaction to the occurrence of an attack may increase the likelihood of further attacks soon after. Further research is needed.

\section{REFERENCES}

l. Uhde TW, Boulenger J-P, Roy-Byrne PP, et al:: Phenomenology and neurobiology of panic disorder, in Tuma AH, Maser JD (eds): Anxiety and the Anxiety Disorders. Hillsdale, NJ, Lawrence Erlbaum Associates, 1985, pp. 557-576

2. Cameron OG, Lee MA, Kotun J, et al.: Circadian symptom fluctuations in people with anxiety disorders. J Affect Disord 11:213-218, 1986

3. Taylor CB, Sheikh J, Agras S, et al.: Ambulatory heart rate changes in patients with panic attacks. Am J Psychiatry 143:478-482, 1986

4. Feller W: Introduction of Probability Theory and Its Applications, 3rd ed., Vol I. New York, Wiley, 1968

5. Special Section: Biological aspects of panic disorders. Am J Psychiatry 143:469-518, 1986

6. Cameron OG, Nesse RM: Systemic hormonal and physiological abnormalities in anxiety disorders: A review. Psychoneuroendocrinology (in press)

7. Doctor RM: Major results of a large-scale pretreatment survey of agoraphobics, in DuPont RL (ed): Phobia: A Comprehensive Survey of Modern Treatments. New York, Brunner/Mazel, 1982, pp. 203-214

8. Kogeorgos J, Fonagy P, Scott DF: Psychiatric symptom patterns of chronic epileptics attending a neurological clinic: A controlled investigation. Br J Psychiatry 140:236-243, 1982

9. Perini G, Mendias R: Depression and anxiety in complex partial seizures. J Nerv Ment Dis 172:287-290, 1984

10. McNamara JO: Kindling model of epilepsy. Adv Neurol 44:303-318, 1986 